\title{
Image Interpolation with Dense Disparity Estimation in Multiview Distributed Video Coding
}

\author{
Wided Miled, Thomas Maugey, Marco Cagnazzo, Béatrice Pesquet-Popescu \\ Télécom ParisTech, TSI departement, 46 rue Barrault 75634 Paris (FRANCE) \\ E-mail: \{miled,maugey,cagnazzo,pesquet\}@ telecom-paristech.fr
}

\begin{abstract}
This paper deals with the side information (SI) generation problem in multiview distributed video coding (MDVC). For inter-view interpolation, we propose a novel dense disparity estimation (DE) approach combined with a popular distributed video coding (DVC) technique. As disparity vectors are computed at the decoder side, and no coding rate is needed to transmit them, dense estimation techniques are made possible, leading to improved results without requiring high coding resources. Experimental results show that the proposed interpolation technique can achieve up to $2.0 \mathrm{~dB}$ improvement in SI reconstruction performance, when compared to state-of-the-art DVC techniques.
\end{abstract}

\section{INTRODUCTION}

Distributed video coding is a new paradigm in video coding, which is receiving a lot of interests nowadays. It is based on Slepian-Wolf and Wyner-Ziv information theoretic results established in the 1970's [1], [2], stating that using dependence only at the decoder can get the same coding performance as conventional source coding. The sources are separately encoded and their statistics exploited at the decoder side. This allows shifting part of the coding complexity from the encoder to the decoder. For multiview video coding, this avoids communication between the different cameras, which is a beneficial characteristic. Many emerging applications, requiring low complexity encoders, could take advantage of the distributed video coding paradigm, such as wireless video sensors for surveillance and mobile camera phones.

However, even though theory was known for a long time, only quite recently efforts have been conducted to apply these theoretical results to practical video coding, first in the field of monoview DVC with the work of Aaron et al. [3] and Girod et al. [4], and then in the field of multiview video [5], [6]. The most popular approach for DVC takes as starting point the Stanford coding scheme [3], the main other being PRISM [7]. The stanford paradigm consists in splitting the input sequence into two subsets, leading to two correlated sources: the key frames (KF) and the Wyner-Ziv frames (WZF). The KFs are encoded and decoded with a regular intra codec, such as H.264 Intra [8]. WZFs, on the other hand, are encoded with a channel coding technique, such as systematic turbo codes, where only the parity bits are sent to the decoder. At the decoder, the key frames are used to generate a prediction of the current WZ frame, called side information (SI), which is fed to the turbo decoder along with the parity bits sent by the encoder. The turbo decoder then uses the parity bits to correct eventual

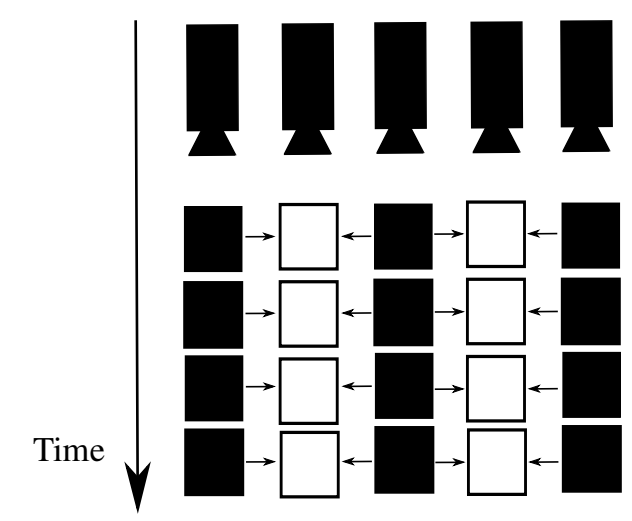

Fig. 1. Time-space frame repartition. KFs are in black and WZFs are in white.

estimation errors between the actual WZF and its SI. In such a framework, DVC performances strongly depend on the quality of the side information. The more accurate the SI is, the fewer bits will be required to encode the WZ frame.

In multiview DVC, SI is generated using either temporal interpolation between the forward and the backward KFs, either inter-view interpolation using KFs from the different views, or a fusion of both interpolations [5], [6]. For temporal interpolation, motion compensated frame interpolation methods are typically used. Many solutions in the literature propose specific methods for inter-view estimation. Ouaret et al. [6] estimate the homographic transformation between the views and use it to build the interpolation. The technique proposed in [5] to perform the inter-camera interpolation requires that a depth map is available for each frame of the Intra cameras. Simpler methods use block-based estimation techniques [9], where disparity vectors are estimated as motion vectors, i.e. assuming that the disparity is blockwise constant and finding the best matching block. However, because this assumption does not always hold and the estimated disparity field does not provide a pixel-to-pixel mapping between left and right views, the interpolated images usually have visible artifacts. While in the classical multiview video coding the cost of transmitting motion and disparity information prevented the expansion of dense estimation methods, in MDVC this information is estimated only at the decoder, and therefore dense fields are not penalized compared with block-based ones. On the contrary, in this way SI interpolation benefits 


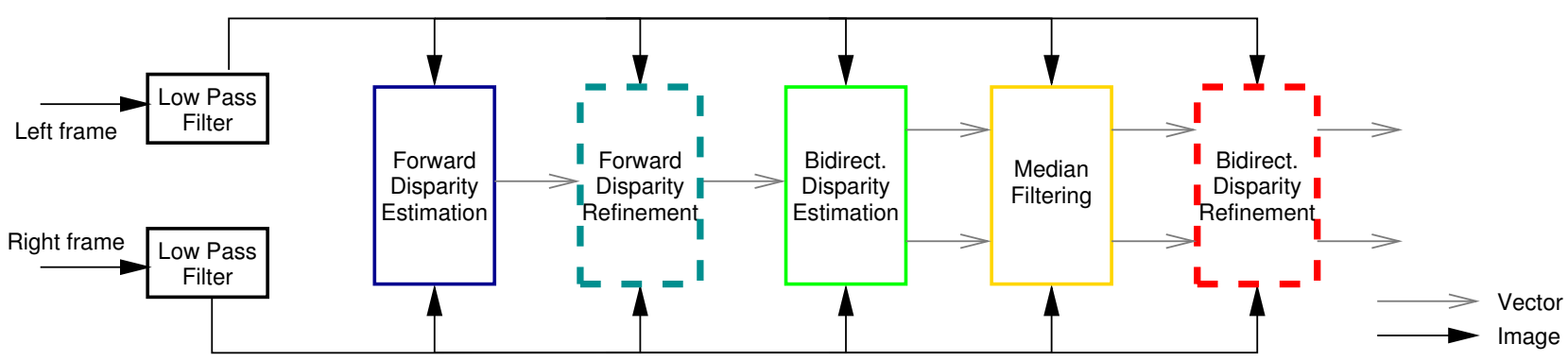

Fig. 2. Proposed image interpolation scheme.

from the increased accuracy of dense displacement vectors without having to pay the cost of an exceedingly high coding rate.

In this paper, we consider a dense disparity estimation approach combined to the popular DISCOVER image interpolation technique [10], [11]. The idea of this combination is to maintain the structure of the DISCOVER scheme and to introduce a refinement stage by using the disparity estimation technique described in [12]. Based on a set theoretic framework, the proposed stereo algorithm minimizes a given objective function subject to various convex constraints, corresponding to a priori information, and yields real valued disparity vectors. To obtain a smooth disparity field while preserving discontinuities, a total variation based regularization constraint is considered.

The remaining of the paper is organized as follows: in Section II, the reference image interpolation DVC scheme and the proposed one are presented. We describe in Section III the employed dense disparity estimation method. Finally, in Section IV, experimental results are given and some conclusions are drawn in Section V.

\section{Reference And Proposed schemes}

We consider the spatiotemporal frame repartition presented in Figure 1 and the case where the Group of Pictures (GOP) size is equal to two. So, only information from adjacent cameras is used to generate an estimation of the current WZF $I_{v}$. Note however that the presented techniques can easily adapt to larger GOP sizes by taking farther frames and recursively using the same structure.

The image interpolation scheme DISCOVER [11], which is an established reference for DVC [13], is depicted in Fig. 3. The inputs are the neighboring left and right KFs, denoted respectively by $I_{v-1}$ and $I_{v+1}$. After a spatial low-pass filtering of the two KFs, a classical forward block matching disparity estimation is performed between them. The resulting monodirectional disparity field is split and used to estimate the forward disparity $\mathbf{v}_{F}$ between $I_{v-1}$ and $I_{v}$, and the backward disparity $\mathbf{v}_{B}$ between $I_{v+1}$ and $I_{v}$. Finally a weighted median filter is then applied on $\mathbf{v}_{F}$ and $\mathbf{v}_{B}$ in order to eliminate the outliers and to get a smooth solution. The WZF estimation is the average between $I_{v-1}$ compensated by $\mathbf{v}_{F}$ and $I_{v+1}$ compensated by $\mathbf{v}_{B}$.
The image interpolation scheme proposed in this work maintains the structure of the DISCOVER scheme, but introduces two new refinement stages (represented by dashed blocks), as illustrated in Figure 2. Depending on the position of the refinement module, the proposed interpolation scheme improves the forward disparity field produced by the mono-directional disparity estimation or the final forward and backward fields $\mathbf{v}_{F}$ and $\mathbf{v}_{B}$ generated by DISCOVER. In what follows, We refer to these two combinations as monodirectional (MR) and bi-directional refinement (BR).

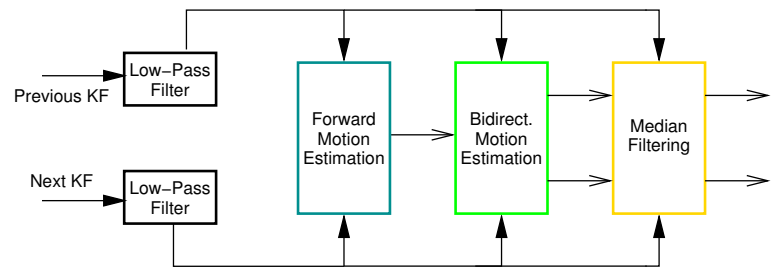

Fig. 3. Discover image interpolation

\section{THE DENSE DISPARITY ESTIMATION ALGORITHM}

To compute the disparity values between two images taken from different viewpoints, the pixels have to undergo a matching procedure, often referred to as the stereo correspondence problem. This process consists in finding for each pixel in one image, its corresponding point in the other image, based on their positions and intensity values. The most critical choice for a stereo matching algorithm is the optimization technique which minimizes a given measure of photometric similarity between pixels.

In the field of dense disparity estimation, global optimization methods have attracted much attention due to their excellent experimental results [14]. These methods exploit various constraints on disparity such as smoothness, view consistency etc., while using efficient and powerful optimization algorithms. In this paper, we consider a disparity estimation approach based on a set theoretic formulation. The proposed method, described in [15], is a global stereo method inspired from recent work developed for image restoration purposes [16]. In the adopted set theoretic framework, the main concern is to find solutions that are consistent with all the available information about the problem. Each piece of information, derived from a prior knowledge and consistency with the 
observed data, is represented by a convex set in the solution space and the intersection of these sets, the feasibility set, constitutes the family of possible solutions. The aim then is to find an acceptable solution minimizing the given objective function. A formulation of this problem in a Hilbert image space $\mathcal{H}$ is therefore:

$$
\text { Find } \mathbf{v} \in S=\bigcap_{i=1}^{m} S_{i} \text { such that } J(\mathbf{v})=\inf J(S),
$$

where the objective $J: \mathcal{H} \rightarrow(-\infty,+\infty]$ is a convex function and the constraint sets $\left(S_{i}\right)_{1 \leq i \leq m}$ are closed convex sets of $\mathcal{H}$. Constraint sets can generally be modelled as level sets:

$$
\forall i \in\{1, \ldots, m\}, \quad S_{i}=\left\{\mathbf{v} \in \mathcal{H} \mid f_{i}(\mathbf{v}) \leq \delta_{i}\right\},
$$

where, for all $i \in\{1, \ldots, m\}, f_{i}: \mathcal{H} \rightarrow \mathbb{R}$ is a continuous convex function and $\left(\delta_{i}\right)_{1 \leq i \leq m}$ are real-valued parameters such that $S=\bigcap_{i=1}^{m} S_{i} \neq \bar{\emptyset}$. Many powerful optimization algorithms have been proposed to solve this convex feasibility problem. For the current work, we employ the constrained quadratic minimization method developed in [17] and particularly well adapted to our needs. However, due to space limitation, we will not describe the algorithm but the reader is referred to [15], [17] for more details.

\section{A. Mono-directional refinement}

The mono-directional refinement stage aims at improving the forward disparity vectors produced by the mono-directional disparity estimation between the left and right KFs $I_{v-1}$ and $I_{v+1}$, using the set theoretic framework described above. For this purpose, we first define the objective function, based on the physical data model. By considering the sum of squared intensity differences (SSD) measure, this objective function can be expressed as follows:

$$
\tilde{J}(\mathbf{v})=\sum_{s \in \mathcal{D}}\left[I_{v+1}(s)-I_{v-1}(s+\mathbf{v}(s))\right]^{2}
$$

where $\mathcal{D} \subset \mathbb{N}^{2}$ is the image support. This expression is nonconvex with respect to the displacement field $\mathbf{v}$. Thus, in order to avoid a non-convex minimization, we use the initial estimate $\overline{\mathbf{v}}$ produced by the first mono-directional estimation stage (based on a block matching process) and we compensate the non-linear term $\left.I_{v-1}(s+\mathbf{v}(s))\right)$ around $\overline{\mathbf{v}}$ using the standard first order approximation:

$$
I_{v-1}(s+\mathbf{v}) \simeq I_{v-1}(s+\overline{\mathbf{v}})+(\mathbf{v}-\overline{\mathbf{v}}) \nabla I_{v-1}(s+\overline{\mathbf{v}}),
$$

where $\nabla I_{v-1}(s+\overline{\mathbf{v}})$ is the gradient of the compensated left frame. Note that for notation concision, we have not made anymore explicit that $\mathbf{v}$ and $\overline{\mathbf{v}}$ are functions of $s$ in the above expression.

With the approximation (4), the cost function $\tilde{J}$ under the minimization in (3) becomes quadratic in $\mathbf{v}$, as follows:

$$
J(\mathbf{v})=\sum_{s \in \mathcal{D}}[L(s) \mathbf{v}(s)-r(s)]^{2}
$$

where

$$
\begin{aligned}
L(s) & =\nabla I_{v-1}(s+\overline{\mathbf{v}}(s)), \\
r(s) & =I_{v+1}(s)-I_{v-1}(s+\overline{\mathbf{v}}(s))+\overline{\mathbf{v}}(s) L(s) .
\end{aligned}
$$

Given the objective function to be minimized, we incorporate, in what follows, the constraints modelling prior information on the estimated disparity field as closed convex sets in the form of Eq. (2). The most common constraint on disparity is the knowledge of its range of possible values. Indeed, disparity values often have known minimal and maximal amplitudes, denoted respectively by $\mathbf{v}_{\min }$ and $\mathbf{v}_{\max }$. The associated set is

$$
S_{1}=\left\{\mathbf{v} \in \mathcal{H} \mid \mathbf{v}_{\min } \leq \mathbf{v} \leq \mathbf{v}_{\max }\right\} .
$$

Furthermore, the disparity map should be smooth in homogeneous areas while keeping sharp edges. This can be achieved with the help of a suitable regularization constraint. In this work, we make use of the total variation (tv) measure which recently emerged as an effective tool to recover smooth images in various image processing research fields. Practically, $\operatorname{tv}(\mathbf{v})$ represents a measure of the lengths of the level lines in the image [18]. Hence, if $\mathbf{v}$ is known a priori to have a certain level of oscillation so that a bound $\tau$ is available on the total variation, controlling $\operatorname{tv}(\mathbf{v})$, restricts the solutions to the convex set

$$
S_{2}=\{\mathbf{v} \in \mathcal{H} \mid \operatorname{tv}(\mathbf{v}) \leq \tau\} .
$$

It should be noted that the upper bound $\tau$ can be estimated with good accuracy from prior experiments and that the considered minimization method is shown to be robust with respect to the choice of this bound [15].

In summary, we formulate the disparity estimation problem as the minimization of the quadratic objective function (8) over the feasibility set $S=\cap_{i=1}^{2} S_{i}$, where the constraint sets $\left(S_{i}\right)_{1 \leq i \leq 2}$ are given by Eq. (6) and (7). The obtained disparity field is then fit into the bidirectional disparity estimation stage to get symmetric disparity predictions from the two KFs. The resulting disparity vector fields are finally filtered with a weighted median filter and used to generate the SI of the current WZF by performing an inter-view interpolation.

\section{B. Bi-directional refinement}

The bi-directional refinement stage consists in recovering first the forward and backward disparity vectors of the DISCOVER algorithm, denoted respectively by $\overline{\mathbf{v}}_{F}$ and $\overline{\mathbf{v}}_{B}$, and applying then the iterative optimization algorithm within the set theoretic framework. The cost function to be minimized, in this case, is based on the assumption that the pixel in the image $I_{v}$ compensated by the forward disparity vector $\mathbf{v}_{F}$ has the same intensity value as the pixel compensated by the backward disparity vector $\mathbf{v}_{B}$. It allows to jointly estimate both disparity vectors, as follows:

$\tilde{J}\left(\mathbf{v}_{B}, \mathbf{v}_{F}\right)=\sum_{s \in \mathcal{D}}\left[I_{v+1}\left(s+\mathbf{v}_{B}(s)\right)-I_{v-1}\left(s+\mathbf{v}_{F}(s)\right)\right]^{2}$.

This expression is non-convex with respect to the displacement fields $\mathbf{v}_{B}$ and $\mathbf{v}_{F}$. Like in the mono-directional refinement 
case, it is approximated by first order approximations to get a convex cost function. However, here we expand both $I_{v+1}$ and $I_{v-1}$ around initial DISCOVER vectors $\overline{\mathbf{v}}_{B}$ and $\overline{\mathbf{v}}_{F}$, respectively:

$$
\begin{aligned}
J\left(\mathbf{v}_{B}, \mathbf{v}_{F}\right) & =\sum_{s \in \mathcal{D}}\left[I_{v+1}\left(s+\overline{\mathbf{v}}_{B}(s)\right)-I_{v-1}\left(s+\overline{\mathbf{v}}_{F}(s)\right)\right. \\
& +\nabla I_{v+1}\left(s+\overline{\mathbf{v}}_{B}(s)\right)\left(\mathbf{v}_{B}(s)-\overline{\mathbf{v}}_{B}(s)\right) \\
& \left.-\nabla I_{v-1}\left(s+\overline{\mathbf{v}}_{F}(s)\right)\left(\mathbf{v}_{F}(s)-\overline{\mathbf{v}}_{F}(s)\right)\right]^{2} \\
& =\sum_{s \in \mathcal{D}}[L(s) \mathbf{v}(s)-r(s)]^{2}
\end{aligned}
$$

where we defined

$$
\begin{aligned}
\mathbf{v} & =\left(\mathbf{v}_{B}, \mathbf{v}_{F}\right)^{\top} \\
L(s) & =\left[\nabla I_{v+1}\left(s+\overline{\mathbf{v}}_{B}(s)\right)-\nabla I_{v-1}\left(s+\overline{\mathbf{v}}_{F}(s)\right)\right] \\
r(s) & =I_{v-1}\left(s+\overline{\mathbf{v}}_{F}(s)\right)-I_{v+1}\left(s+\overline{\mathbf{v}}_{B}(s)\right)+L(s)\left(\overline{\mathbf{v}}_{B}, \overline{\mathbf{v}}_{F}\right)^{\top} .
\end{aligned}
$$

Once the global convex objective function to be minimized is defined, we consider now convex constraints based on the properties of the estimated fields. We retain, as previously, the range values constraint and the edge preserving regularization one. The constraint sets associated with the first a priori information are

$$
\begin{aligned}
& S_{1}=\left\{\mathbf{v} \in \mathcal{H} \mid \mathbf{v}_{\min }^{B} \leq \mathbf{v}_{B} \leq \mathbf{v}_{\max }^{B}\right\}, \\
& S_{2}=\left\{\mathbf{v} \in \mathcal{H} \mid \mathbf{v}_{\min }^{F} \leq \mathbf{v}_{F} \leq \mathbf{v}_{\max }^{F}\right\} .
\end{aligned}
$$

The regularization constraint, whose effect is to smooth homogenous regions in the disparity field while preserving edges, introduces a bound on the integral of the norm of the spatial gradient. Thus, imposing an upper bound on the total variation allows to efficiently restrict the solution to the constraint sets:

$$
\begin{gathered}
S_{3}=\left\{\mathbf{v} \in \mathcal{H} \mid \operatorname{tv}\left(\mathbf{v}_{B}\right) \leq \tau_{\mathbf{v}_{B}}\right\}, \\
S_{4}=\left\{\mathbf{v} \in \mathcal{H} \mid \operatorname{tv}\left(\mathbf{v}_{F}\right) \leq \tau_{\mathbf{v}_{F}}\right\},
\end{gathered}
$$

where $\tau_{\mathbf{v}_{B}}$ and $\tau_{\mathbf{v}_{F}}$ are positive constants that can be estimated from prior experiments and image databases.

The problem of disparity estimation can finally be formulated as jointly finding the forward and backward disparity fields which minimize the energy function (9) subject to the constraints $\left(S_{i}\right)_{1 \leq i \leq 4}$. The problem becomes therefore bivariate and to solve it, we have adapted the convex optimization algorithm considered in the mono-directional case, taking into account the dimensionality of the problem.

\section{EXPERIMENTAL RESULTS}

We evaluate the proposed method on the two multiview rectified test sequences "Book Arrival" and "Outdoor", for which the original first frames of view 2 are shown in Figures 4(a) and 4(b), respectively. For both sequences, the spatial resolution has been halved by two, so that the images have size $512 \times 386$, and only the first 7 cameras were used. The monodirectional and bi-directional refinement techniques have been performed to estimate the SI of the WZFs corresponding to views 2, 4 and 6. For each view, we consider four quantization steps $(\mathrm{QP}=31,34,36$ and 40$)$, in order to observe the behavior

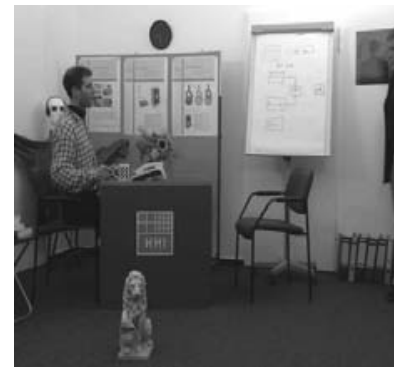

(a)

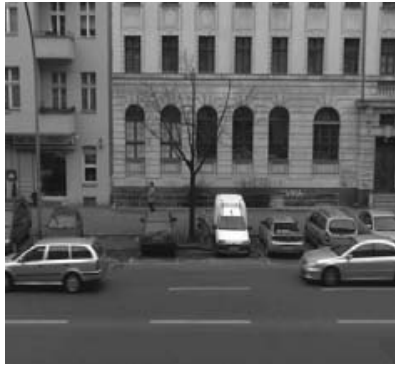

(b)
Fig. 4. Reference first frame for (a) "Book Arrival" and (b) "Outdoor" multiview test sequences.

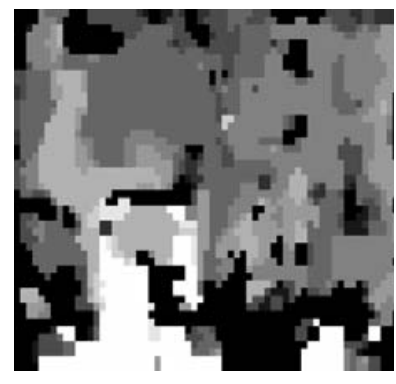

(a) DISCOVER

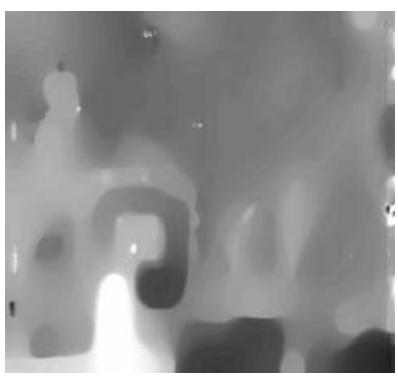

(b) MR technique
Fig. 5. Horizontal disparity fields for the "Book Arrival" test sequence.

of both refinement techniques, compared to the DISCOVER algorithm, in a relatively wide range of bit-rates. The inspection of the horizontal disparity field obtained with the DISCOVER algorithm and the proposed MR technique allows a first qualitative comparison between the two methods. We show in Figure 5 the results: as we can see, the proposed technique produces a smooth disparity field and is more robust to noise with respect to the reference.

Of course, a quantitative comparison of the two method is necessary. The parameter considered at this end is the PSNR

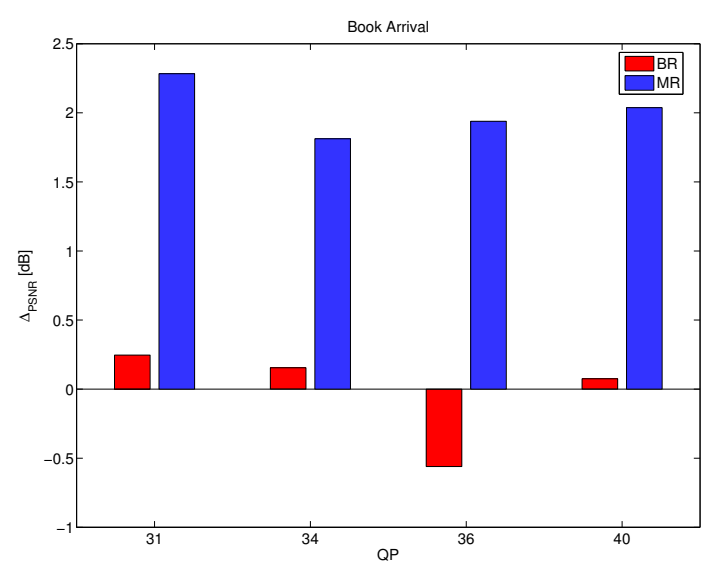

Fig. 6. SI PSNR improvement $[\mathrm{dB}]$ between reference and proposed MR and BR techniques for the "Book Arrival" sequence. 
TABLE I

COMPARATIVE RESULTS USING THE PSNR OF SI IMAGES FOR DIFFERENT QPS.

\begin{tabular}{|c|c|c|c|c|c|c|c|c|}
\hline \multirow[b]{2}{*}{ Sequence } & \multicolumn{4}{|c|}{ DISCOVER } & \multicolumn{4}{|c|}{ Proposed MR } \\
\hline & 31 & 34 & 36 & 40 & 31 & 34 & 36 & 40 \\
\hline Book Arrival & 31.97 & 31.63 & 30.93 & 29.13 & 34.25 & 33.45 & 32.87 & 31.17 \\
\hline Outdoor & 35.39 & 33.78 & 32.62 & 30.18 & 35.63 & 34.03 & 32.85 & 30.38 \\
\hline
\end{tabular}

between the original WZF and its estimation produced by each of the compared techniques. In particular we computed the averaged PSNR over three views for each test sequence. The results for the "Book Arrival" sequence are illustrated in Figure 6. We observe that the proposed MR technique is able to improve the WZF quality by up to $2.0 \mathrm{~dB}$ with respect to the DISCOVER algorithm. The BR technique achieves, however, only up to $0.2 \mathrm{~dB}$ for some quantization steps, and even degrades the result for $\mathrm{QP}=36$. Moreover, it is important to note that this technique presents a higher computational cost than the MR one, due to the fact that two dense fields have to be recovered simultaneously. The same results have been observed for the "Outdoor" sequence. This is why we decide in what follows to consider only the results of the MR method. Figure 7 shows the results of the "Outdoor" sequence, for which the MR technique improves the WZF quality up to $0.25 \mathrm{~dB}$. The smaller gain obtained for this sequence, with respect to the one obtained for the "Book Arrival" sequence, can be justified by the fact that for the "Outdoor" sequence, as the range of disparity values is small, the DISCOVER algorithm already obtains good results, and so the refinement stage cannot give significant gain. The PSNR values for both sequences and each QP are reported in Table IV. Furthermore, Figure 8 illustrates a zoom applied on the SI reconstructed image for the "Book Arrival" multiview sequence. The proposed MR technique provides a better quality of the SI estimation than the reference algorithm which yields visible artifacts. The overall results confirm that the proposed technique produces better image interpolation than the reference. Concerning the computational complexity of the dense disparity estimation algorithm, our current implementation, which is completely written in Matlab code, has a running time of about $3.5 \mathrm{~min}$ (using a Pentium $2.6 \mathrm{GHz}$ PC). It must be mentioned that more efficient implementations of this algorithm can be written in C. In addition, due to its iterative structure, this algorithm is well suited for an implementation on parallel processors which, if exploited, can help in reducing the computational time.

\section{CONCLUSION}

In this work, we have presented the benefits of using a dense disparity field in the MDVC framework. While in the classical video coding paradigm, a dense field suffers from an exceeding coding cost and computational complexity, in MDVC we can obtain good quality dense fields at the decoder side, yielding better SI reconstruction. The dense estimation method considered in this paper is based on a global optimization algorithm which minimizes a quadratic convex objective function subject to some appropriate convex constraints. This

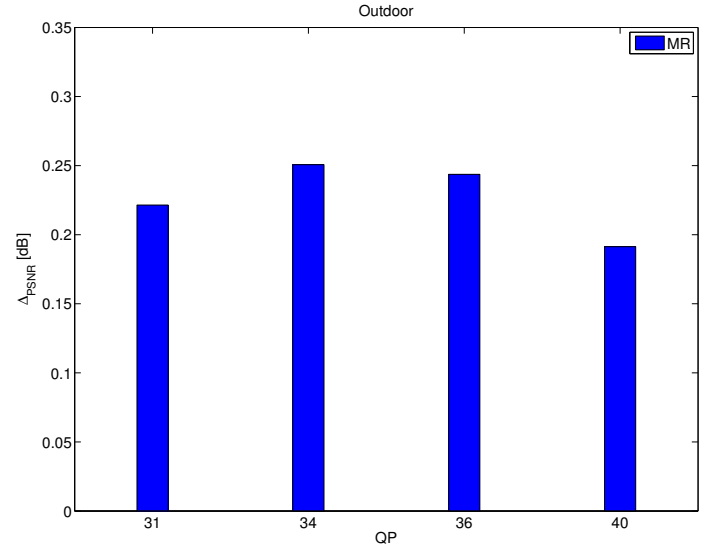

Fig. 7. SI PSNR improvement $[\mathrm{dB}]$ between reference and proposed $\mathrm{MR}$ technique for the "Outdoor" sequence.

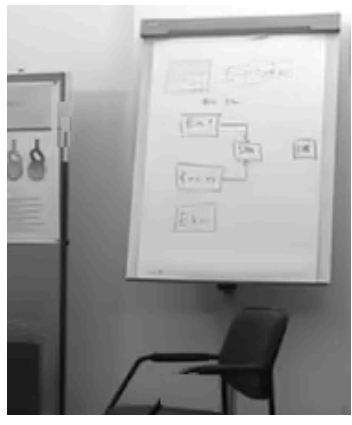

(a)

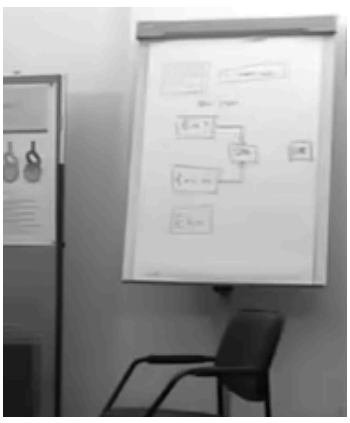

(b)
Fig. 8. SI reconstruction for "Book Arrival" using (a) DISCOVER and (b) MR techniques.

method was combined to the popular DISCOVER algorithm, which actually acts as an initialization of the proposed iterative algorithm. Using the dense estimation refinement in the early stage of the DISCOVER scheme allows us to improve the SI quality of up to $2.0 \mathrm{~dB}$. The results are encouraging and future studies will be conducted on further application of the dense estimation, for exploiting both temporal and inter-views correlations in MDVC. The quality improvement relating to the GOP size between generated SI will be also studied.

\section{REFERENCES}

[1] D. Slepian and J. K. Wolf, "Noiseless Coding of Correlated Information Sources," IEEE Trans. on Information Theory, vol. 19, pp. 471-480, July 1973.

[2] A. Wyner and J. Ziv, "The Rate-Distortion Function for Source Coding with Side Information at the Receiver," IEEE Trans. on Information Theory, vol. 22, pp. 1-11, Jan. 1976. 
[3] A. Aaron, R. Zhang and B. Girod, "Wyner-Ziv coding of motion video," in Proc. Asilomar Conference on Signals and Systems, Pacific Grove, California, Nov. 2002.

[4] B. Girod, A. Aaron, S. Rane and D. Rebollo-Monedero, "Distributed Video Coding", Proc. of the IEEE, vol. 93, no. 71, pp. 71-83, Jan. 2005.

[5] X. Artigas, E. Angeli and L. Torres, "Side information generation for multiview distributed video coding using a fusion approach," 7th Nordic Signal Processing Symposium, Iceland, June 2006.

[6] M. Ouaret, F. Dufaux, and T. Ebrahimi, "Fusion-based multiview distributed video coding," ACM International Workshop on Video Surveillance and Sensor Networks, Santa Barbara, California, USA, Oct. 2006.

[7] R. Puri and K. Ramchandran, "PRISM: A video coding architecture based on distribued compression principles," Tech. Rep. UCB/ERL M03/6, EECS Departement, University of California, Berkeley, 2003.

[8] Advanced video coding for generic audiovisual services, ITU-T Rec. H.264 and ISO/IEC 14496-10 (MPEG-4 AVC), Version 1: May 2003, Version 8: Consented in July 2007.

[9] J. D. Areia, J. Ascenso, C. Brites and F. Pereira, "Wyner-Ziv Stereo Video Coding using a Side Information Fusion Approach," IEEE International Workshop on Multimedia Signal Processing, Chania, Greece, Oct. 2007, pp. $453-456$.

[10] J. Ascenso, C. Brites and F. Pereira, "Improving Frame Interpolation with Spatial Motion Smoothing for Pixel Domain Distributed Video Coding," EURASIP Conference on Speech and Image Processing, Multimedia Communications and Services, Smolenice, Slovak Republic, June 2005.

[11] C. Guillemot, F. Pereira, L. Torres, T. Ebrahimi, R. Leonardi and J. Ostermann, "Distributed Monoview and Multiview Video Coding: Basics, Problems and Recent Advances," IEEE Signal Processing Magazine, Special Issue on Signal Processing for Multiterminal Communication Systems, Sep. 2007, pp. 7-76.

[12] W. Miled, J.-C. Pesquet and M. Parent, "A Convex optimization approach for depth estimation under illumination variation," IEEE Transactions on Image Processing, vol. 18, no. 4, Apr. 2009, pp. 813-830.

[13] M. Cagnazzo, T. Maugey and B. Pesquet-Popescu, "A differential motion estimation method for image interpolation in distributed video coding," in Proceed. of IEEE Intern. Conf. Acoust. Speech and Sign. Process., Taipei, Taiwan, Apr. 2009.

[14] D. Scharstein and R. Szeliski, "A Taxonomy and evaluation of dense two-frame stereo correspondence algorithms," Int. Journal of Computer vision, vol. 47, pp. 7-42, 2002.

[15] W. Miled, J.-C. Pesquet and M. Parent, "Disparity map estimation using a total variation bound," Proc. 3rd Canadian Conf. Comput. Robot Vis., Quebec, Canada, June 2006, pp. 48-55.

[16] P.L. Combettes, "A block iterative Surrogate Constraint Splitting Method for quadratic Signal recovery," IEEE Trans. Signal Process., vol. 51, no. 7, pp.1771-1782, July 2003.

[17] P.L. Combettes and J.C. Pesquet, "Image restoration subject to a total variation constraint,"IEEE Trans. Image Proc., Vol. 13, pp. 1213-1222, Sept. 2004.

[18] L.I. Rudin, S. Osher and E. Fatemi, "Nonlinear total variation based noise removal algorithms," Physica D, vol. 60, pp. 259-268, 1992. 